\title{
Monotone Measures Defined by Pan-Integral
}

\author{
Peipei Wang1, Minhao $\mathrm{Yu}^{2}$, Jun $\mathrm{Li}^{2 *}$ \\ ${ }^{1}$ Property and Finance Department, National Museum of China, Beijing, China \\ ${ }^{2}$ School of Sciences, Communication University of China, Beijing, China \\ Email: gloriapeipeiwang@outlook.com,yuminhao@cuc.edu.cn, *lijun@cuc.edu.cn
}

How to cite this paper: Wang, P.P., Yu, M.H. and Li, J. (2018) Monotone Measures Defined by Pan-Integral. Advances in Pure Mathematics, 8, 535-547.

https://doi.org/10.4236/apm.2018.86031

Received: April 29, 2018

Accepted: June 17, 2018

Published: June 20, 2018

Copyright (C) 2018 by authors and Scientific Research Publishing Inc. This work is licensed under the Creative Commons Attribution International License (CC BY 4.0).

http://creativecommons.org/licenses/by/4.0/

\section{(c) ${ }_{\mathrm{EY}}$ Open Access}

\begin{abstract}
Given a pan-space $\left(X, \mathcal{A}, \mu, \bar{R}_{+}, \oplus, \otimes\right)$ and a nonnegative measurable function $f$ on measurable space $(X, \mathcal{A})$, the pan-integral of $f$ with respect to monotone measure $\mu$ and pan-operation $(\oplus, \otimes)$ determines a new monotone measure $\lambda_{f}^{(\oplus, \otimes)}$ on $(X, \mathcal{A})$. Such the new monotone measure $\lambda_{f}^{(\oplus, \otimes)}$ is absolutely continuous with respect to the monotone measure $\mu$. We show that the new monotone measure preserves some important structural characteristics of the original monotone measures, such as continuity from below, subadditivity, null-additivity, weak null-additivity and (S) property. Since the pan-integral based on a pair of pan-operations $(\oplus, \otimes)$ covers the Sugeno integral (based on $(\vee, \wedge)$ ) and the Shilkret integral (based on $(\vee, \cdot)$ ), therefore, the previous related results for the Sugeno integral are covered by the results presented here, in the meantime, some special results related the Shilkret integral are also obtained.
\end{abstract}

\section{Keywords}

Monotone Measure, Pan-Integral, Sugeno Integral, Choquet Integral, Shilkret Integral

\section{Introduction}

In generalized measure and integral theory, there are several kinds of important nonlinear integrals, the Choquet integral [1], the Sugeno integral [2], the pan-integral [3] and the concave integral introduced by Lehrer [4], etc. (see also [5]). The pan-integral with respect to monotone measure $\mu$ relates to a commutative isotonic semiring $\left(\bar{R}_{+}, \oplus, \otimes\right)$, where $\oplus$ is a pan-addition and $\otimes$ is a pan-multiplication related by the distributivity property (see also [6]). This integral generalizes the Lebesgue integral and the Sugeno integral. When consi- 
dering a $\sigma$-additive measure $m$ and the commutative isotonic semiring $\left(\bar{R}_{+},+, \cdot\right)$, the Lebesgue integral coincides with the pan-integral; when considering a monotone measure $\mu$ and the commutative isotonic semiring $\left(\bar{R}_{+}, \vee, \wedge\right)$, the Sugeno integral is recovered by the pan-integral with respect to $(\vee, \wedge)$. The more details on the pan-integrals can be also found in [6]-[12].

In classical measure theory [13], given measure space $(X, \mathcal{A}, m)$ and a nonnegative measurable function $f$ on $(X, \mathcal{A})$, the set function $v_{f}$, defined by the Lebesgue integral of $f$,

$$
v_{f}(A)=\int_{A} f \mathrm{~d} m, \quad \forall A \in \mathcal{A} .
$$

is a measure, i.e., $v_{f}$ is $\sigma$-additive and $v_{f}(\varnothing)=0$, and $v_{f}$ is absolutely continuous with respect to $m$.

For the Sugeno integral and the Choquet integral, the issue above has also been discussed respectively in [14] and [15]. Given a measurable space $(X, \mathcal{A})$, a monotone measure $\mu$, and a nonnegative measurable function $f$ on $(X, \mathcal{A})$, the Sugeno integral (or the Choquet integral) with respect to $\mu$ determines a new monotone measure $\lambda_{f}^{(S u)}$ (or $\lambda_{f}^{(C h)}$ ). In general the monotone measures lose additivity, and they appear to be much looser than the classical measures. This new construction raises an important question: Do structural features of the original monotone measure $\mu$ still hold for these new monotone measures? It has been shown that the new monotone measures inherit almost all desirable structural characteristics of $\mu$, such as, subadditivity, continuity from below, continuity from above, null-additivity, weak null-additivity and autocontinuity (see [14] [15] [16]).

In this paper we discuss the above-mentioned issue for the pan-integrals. Given a pan-space $\left(X, \mathcal{A}, \mu, \bar{R}_{+}, \oplus, \otimes\right)$ and a nonnegative measurable function $f$ on $(X, \mathcal{A})$, the pan-integral of $f$ with respect to $\mu$ and pan-operation $(\oplus, \otimes)$ determines a new monotone measure $\lambda_{f}^{(\oplus, \otimes)}$ on $(X, \mathcal{A})$. Such the monotone measure $\lambda_{f}^{(\oplus, \otimes)}$ is absolutely continuous with respect to $\mu$. We are going to show that $\lambda_{f}^{(\oplus, \otimes)}$ reserves some important structural characteristics of $\mu$, which is similar to the cases of the Choquet integral and the Sugeno integral. We are also going to show that if monotone measure is subadditive, then the pan-integral with respect to the monotone measure is a monotone superadditive functional. Since the pan-integral covers the Sugeno integral, while the Shilkret integral, the previous related results for the Sugeno integral become special cases of the results presented here, in the meantime, some special results related the Shilkret integral are shown.

\section{Preliminaries}

Let $X$ be a non-empty set, $\mathcal{A}$ a $\sigma$-algebra of subsets of $X$. As usual the pair $(X, \mathcal{A})$ denotes a measurable space. Denotes $R_{+}=[0,+\infty), \bar{R}_{+}=[0,+\infty]$. Unless stated otherwise, all subsets mentioned are supposed to belong to $\mathcal{A}$.

Definition 1. ([2]) An extended real valued set function $\mu: \mathcal{A} \rightarrow \bar{R}_{+}$is 
called a monotone measure defined on $(X, \mathcal{A})$, if $\mu$ satisfies the following two conditions:

1) $\mu(\varnothing)=0$ and $\mu(X)>0$;

(vanishing at $\varnothing$ )

2) $\forall P, Q \in \mathcal{A}, P \subset Q$ implies $\mu(P) \leq \mu(Q)$.

(monotonicity)

When $\mu$ is a monotone measure, the triple $(X, \mathcal{A}, \mu)$ is called a monotone measure space ([10]).

In many literature, a monotone measure is also known as "non-additive measure", "capacity", "fuzzy measure", "non-additive measure" or "non-additive probability", etc. (see [1] [6] [17] [18] [19]).

In this paper we restrict our discussion on fixed measurable space $(X, \mathcal{A})$. Let $\mathcal{M}^{(X, \mathcal{A})}$ the set of all monotone measures defined on $(X, \mathcal{A})$.

A monotone measure $\mu: A \rightarrow \bar{R}_{+}$is said to be: continuous from below [20], if $\lim _{n \rightarrow \infty} \mu\left(P_{n}\right)=\mu(P)$ whenever $P_{n} \nearrow P$; continuous from above [20], if $\lim _{n \rightarrow \infty} \mu\left(Q_{n}\right)=\mu(Q)$ whenever $Q_{n} \searrow Q$ and $\mu\left(Q_{1}\right)<\infty$; continuous, if it is continuous both from below and from above; subadditive [20], if $\mu(P \cup Q) \leq \mu(P)+\mu(Q)$ whenever $P, Q \in \mathcal{A}$; superadditive, if $\mu(P \cup Q) \geq \mu(P)+\mu(Q)$ whenever $P, Q \in \mathcal{A}, P \cap Q=\varnothing$; null-additive ([6]), if $\mu(A \cup N)=\mu(A)$ for any $A$ whenever $\mu(N)=0$; weakly null-additive [6], if $\mu(R \cup S)=0$, for any $R, S \in A$ such that $\mu(R)=\mu(S)=0$.

Obviously, the subadditivity of $\mu$ implies the null-additivity, and the later implies weak null-additivity. The converse implications may not be true.

Definition 2 ([21] [22]) Let $\mu, v \in \mathcal{M}^{(X, A)}$. We say that

1) $\mu$ is absolutely continuous of Type $I$ with respect to $v$, denoted by $\mu \ll_{I} v$, iff $\mu(A)=0$ whenever $v(A)=0$;

2) $\mu$ is absolutely continuous of Type $V I$ with respect to $v$, denoted by $\mu \ll_{V I} v$, iff $\mu\left(A_{n}\right) \rightarrow 0(n \rightarrow \infty)$ whenever $v\left(A_{n}\right) \rightarrow 0(n \rightarrow \infty)$.

Obviously, $\mu \ll_{V I} v$ implies $\mu \ll_{I} v$. The inverse statement may not be true.

\section{Pan-Operation and Integrals}

In [3] Yang introduced the concept of pan-integral (see also [6] and [12]), which is a type of nonlinear integral with respect to fuzzy measure. These integrals involve two binary operations, the pan-addition $\oplus$ and pan-multiplication $\otimes$ of real numbers. We recall a commutative isotonic semiring [6] (see also [8] [10] [11] [17] [23]).

Definition 3. A binary operation $\oplus$ on $\bar{R}_{+}$is called a pseudo-addition on $\bar{R}_{+}$if and only if it satisfies the following requirements:

(PA1) $r \oplus s=s \oplus r$;

(PA2) $(r \oplus s) \oplus t=r \oplus(s \oplus t)$;

(PA3) $r \leq s \Rightarrow r \oplus t \leq s \oplus t$ for any $t$;

(PA4) $r \oplus 0=r$;

(PA5) $r_{n} \rightarrow r$ and $s_{n} \rightarrow s \Rightarrow r_{n} \oplus s_{n} \rightarrow r \oplus s$.

From associativity (PA2), we may write $r_{1} \oplus r_{2} \oplus \cdots \oplus r_{n}$ as $\bigoplus_{i=1}^{n} r_{i}$, and denote 
$\bigoplus_{i=1}^{\infty} r_{i}=\lim _{n \rightarrow \infty} \bigoplus_{i=1}^{n} r_{i}$

Definition 4. A binary operation $\otimes$ on $\bar{R}_{+}$is called a pseudo-multiplication (with respect to pseudo-addition $\oplus$ ) on $\bar{R}_{+}$if and only if it fulfills the following conditions:

$(\mathrm{PM} 1) \quad r \otimes s=s \otimes r$;

$(\mathrm{PM} 2)(r \otimes s) \otimes t=r \otimes(s \otimes t)$;

(PM3) $r \otimes(s \oplus t)=(r \otimes s) \oplus(r \otimes t)$;

(PM4) $r \leq s \Rightarrow r \otimes t \leq s \otimes t$ for any $t$;

(PM5) $r \otimes s=0 \Leftrightarrow r=0$ or $s=0$;

(PM6) there exists $e \in \bar{R}_{+}$, which is called the unit element, such that $e \otimes r=r$ for any $r \in \bar{R}_{+}$;

(PM7) $r, s \in(0,+\infty), \quad r_{n} \rightarrow r$ and $r_{n} \rightarrow r \Rightarrow r_{n} \otimes s_{n} \rightarrow r \otimes s$.

When $\oplus$ is a pseudo-addition on $\bar{R}_{+}$and $\oplus$ is a pseudo-multiplication (with respect to $\oplus$ ) on $\bar{R}_{+}$, the triple $\left(\bar{R}_{+}, \oplus, \otimes\right)$ is called a commutative isotonic semiring on $\bar{R}_{+}([6])$, and $\left(X, \mathcal{A}, \mu, \bar{R}_{+}, \oplus, \otimes\right)$ is called a pan-space ([6]).

Next we recall the concept of pan-integral. $\mathcal{F}_{+}^{(X, \mathcal{A})}$ denotes the set of all finite nonnegative $\mathcal{A}$-measurable functions on $X$. A finite partition of $A \in \mathcal{A}$ is a finite disjoint system of sets $\left\{A_{i}\right\}_{i=1}^{n} \subset \mathcal{A}$ such that $A_{i} \cap A_{j}=\varnothing$ for $i \neq j$ and $\bigcup_{i=1}^{n} A_{i}=A$.

Definition 5. ([3] [6]) Let $\left(X, \mathcal{A}, \mu, \bar{R}_{+}, \oplus, \otimes\right)$ be a pan-space. For any $f \in \mathcal{F}_{+}^{(X, \mathcal{A})}, A \in \mathcal{A}$, the pan-integral of fover $A$ with respect to $\mu$, is defined by

$$
\int_{A}^{(\oplus, \otimes)} f \mathrm{~d} \mu=\sup _{\mathcal{E} \in \hat{\mathcal{P}}}\left\{\bigoplus_{E \in \mathcal{E}}\left[\left(\inf _{x \in A \cap E} f(x)\right) \otimes \mu(A \cap E)\right]\right\},
$$

where $\hat{\mathcal{P}}$ is the set of all finite partitions of $X$.

When $A=X, \int_{X}^{(\oplus, \otimes)} f \mathrm{~d} \mu$ is written as $\int^{(\oplus, \otimes)} f \mathrm{~d} \mu$.

The pan-integral of $f$ over $A$ with respect to $\mu$ can be expressed by pan-simple functions, as follows:

$$
\int_{A}^{(\oplus, \otimes)} f \mathrm{~d} \mu=\sup \left\{\bigoplus_{i=1}^{n}\left(\lambda_{i} \otimes \mu\left(A_{i}\right)\right): \bigoplus_{i=1}^{n}\left(\lambda_{i} \otimes \chi_{A_{i}}\right) \leq f, \lambda_{i} \geq 0,\left\{A_{i}\right\}_{i=1}^{n} \in \hat{\mathcal{P}}_{A}\right\},
$$

where $\chi_{A}$ is the pan-characteristic function of $A$, i.e.,

$$
\chi_{A}(x)= \begin{cases}e & \text { if } x \in A \\ 0 & \text { otherwise }\end{cases}
$$

$\bigoplus_{i=1}^{n}\left(\lambda_{i} \otimes \mu\left(A_{i}\right)\right)$ is pan-simple function ([6]) and $\hat{\mathcal{P}}_{A}$ is the set of all finite partitions of $A$.

Note. A related concept of generalized Lebesgue integral based on a generalized ring $\left(\bar{R}_{+}, \oplus, \otimes\right)$ was proposed and discussed (see [12]).

The following is some basic properties of the pan-integrals (see [6] [12]).

Proposition 6. Let $f, g \in \mathcal{F}_{+}^{(X, \mathcal{A})}$ and $a \in R_{+}$. Then we have the following:

1) if $\mu(A)=0$, then $\int_{A}^{(\oplus, \otimes)} f \mathrm{~d} \mu=0$; 
2) if $f=0$ a.e. $[\mu]$ on $A$, i.e., $\mu(\{f \neq 0\} \cap A)=0$, then $\int_{A}^{(\oplus, \otimes)} f \mathrm{~d} \mu=0$. Further, if $\mu$ is continuous from below, then $\int_{A}^{(\oplus, \otimes)} f \mathrm{~d} \mu=0$ if and only if $f=0$ a.e. $[\mu]$ on $A$;

3) $\int_{A}^{(\oplus, \otimes)} f \mathrm{~d} \mu=\int^{(\oplus, \otimes)} f \otimes \chi_{A} \mathrm{~d} \mu$;

4) if $f \leq g$, then $\int_{A}^{(\oplus, \otimes)} f \mathrm{~d} \mu \leq \int_{A}^{(\oplus, \otimes)} g \mathrm{~d} \mu$;

5) if $A \subset B$, then $\int_{A}^{(\oplus, \otimes)} f \mathrm{~d} \mu \leq \int_{B}^{(\oplus, \otimes)} f \mathrm{~d} \mu$

6) $\int^{(\oplus, \otimes)} a \otimes f \mathrm{~d} \mu \geq a \otimes \int^{(\oplus, \otimes)} f \mathrm{~d} \mu$. In particular, for $A \in \mathcal{A}$,

$$
\int^{(\oplus, \otimes)} a \otimes \chi_{A} \mathrm{~d} \mu \geq a \otimes \int^{(\oplus, \otimes)} \chi_{A} \mathrm{~d} \mu .
$$

The following is the Monotone Convergence Theorem of the pan-integral ([6] [12]).

Proposition 7. Let $\mu$ be continuous from below, $A \in \mathcal{A}$, $f_{n}, f \in \mathcal{F}_{+}^{(X, \mathcal{A})}, n=1,2, \cdots$. If $f_{n} \nearrow f(n \rightarrow \infty)$, then

$$
\int_{A}^{(\oplus, \otimes)} f \mathrm{~d} \mu=\lim _{n \rightarrow \infty} \int_{A}^{(\oplus, \otimes)} f_{n} \mathrm{~d} \mu \text {. }
$$

Proposition 8. Let $f, g \in \mathcal{F}_{+}^{(X, \mathcal{A})}$. We have the following:

1) $\int^{(\oplus, \otimes)} f \mathrm{~d} \mu=\int_{\{f>0\}}^{(\oplus, \otimes)} f \mathrm{~d} \mu$, where $\{f>0\}=\{x \in X: f(x)>0\}$;

2) If $\mu$ is null-additive and $f=g$ a.e. $[\mu]$ on $A$, i.e., $\mu(\{f \neq g\} \cap A)=0$, then

$$
\int_{A}^{(\oplus, \otimes)} f \mathrm{~d} \mu=\int_{A}^{(\oplus, \otimes)} g \mathrm{~d} \mu .
$$

Proof. Noting that $f=f \otimes \chi_{\{f>0\}}$, from Proposition 63 ), we can get 1).

2). Let $A_{1}=\{x \in A, f(x) \neq g(x)\}$, then $\mu\left(A_{1}\right)=0$ and for any subset $B$ of $A$ we have $\mu(B)=\mu\left(B \backslash A_{1}\right)$. Thus, for any $\oplus_{i=1}^{n}\left[\lambda_{i} \otimes \chi_{B_{i}}\right] \leq f(x) \otimes \chi_{A}$, $\oplus_{i=1}^{n}\left[\lambda_{i} \otimes \chi_{B_{i} \backslash A_{1}}\right] \leq g(x) \otimes \chi_{A}$. Moreover,

$$
\int_{A}^{(\oplus, \otimes)} g \mathrm{~d} \mu \geq \bigoplus_{i=1}^{n}\left[\lambda_{i} \otimes \mu\left(B_{i} \backslash A_{1}\right)\right]=\bigoplus_{i=1}^{n}\left[\lambda_{i} \otimes \mu\left(B_{i}\right)\right],
$$

which implies that $\int_{A}^{(\oplus, \otimes)} g \mathrm{~d} \mu \geq \int_{A}^{(\oplus, \otimes)} f \mathrm{~d} \mu$. In a similar way, we have $\int_{A}^{(\oplus, \otimes)} f \mathrm{~d} \mu \geq \int_{A}^{(\oplus, \otimes)} g \mathrm{~d} \mu$, and thus 2) holds.

The following is three kinds of important nonlinear integrals [1] [2] [24], see also [5]. Consider a nonnegative real-valued measurable function $f \in \mathcal{F}_{+}^{(X, \mathcal{A})}$ and $\mu \in \mathcal{M}^{(X, \mathcal{A})}$, i.e., $(\mu, f) \in \mathcal{M}^{(X, \mathcal{A})} \times \mathcal{F}_{+}^{(X, \mathcal{A})}$.

The Choquet integral [1] of $f$ on $X$ with respect to $\mu$, denoted by $(C h) \int f \mathrm{~d} \mu$, is defined by

$$
(C h) \int f \mathrm{~d} \mu=\int_{0}^{\infty} \mu(\{x: f(x) \geq t\}) \mathrm{d} t
$$

where the right side integral is Riemann integral.

The Sugeno integral [2] of $f$ on $X$ with respect to $\mu$, denoted by $(S) \int f \mathrm{~d} \mu$, is defined by 


$$
S(u) \int f \mathrm{~d} \mu=\sup _{0 \leq t<+\infty}[t \wedge \mu(\{x: f(x) \geq t\})]
$$

The Shilkret integral [24] of $f$ on $X$ with respect to $\mu$, is defined by

$$
(S h) \int f \mathrm{~d} \mu=\sup _{0 \leq t<+\infty}[t \cdot \mu(\{x: f(x) \geq t\})]
$$

For $f \in \mathcal{F}_{+}^{(X, \mathcal{A})}$, if $(C h) \int f \mathrm{~d} \mu<\infty$ (resp. $\left.(S u) \int f \mathrm{~d} \mu<\infty\right)$, we say that $f$ is Choquet integrable (resp. Sugeno integrable).

Note that in the case of commutative isotonic semiring $\left(\bar{R}_{+}, \vee, \wedge\right)$, the Sugeno integral is recovered, while for $\left(\bar{R}_{+}, \vee, \cdot\right)$, the Shilkret integral is covered by the pan-integral in Definition 5, i.e.,

$$
(S u) \int f \mathrm{~d} \mu=\int f \mathrm{~d} \mu
$$

and

$$
(S h) \int f \mathrm{~d} \mu=\int f \mathrm{~d} \mu .
$$

\section{Monotone Measures Defined by Pan-Integral}

Given a pan-space $\left(X, \mathcal{A}, \mu, \bar{R}_{+}, \oplus, \otimes\right)$ and $f \in \mathcal{F}_{+}^{(X, \mathcal{A})}$, we define a new set function $\lambda_{f}^{(\oplus, \otimes)}$ by

$$
\lambda_{f}^{(\oplus, \otimes)}(A)=\int_{A}^{(\oplus, \otimes)} f \mathrm{~d} \mu, \forall A \in \mathcal{A} .
$$

From Definition 5 and Proposition $6(\mathrm{v})$, we know that $\lambda_{f}^{(\oplus, \otimes)}$ is a monotone measure on $(X, \mathcal{A})$, too, i.e., $\lambda_{f}^{(\oplus, \otimes)} \in \mathcal{M}^{(X, \mathcal{A})}$.

The new monotone measure $\lambda_{f}^{(\oplus, \otimes)}$ is said to be monotone measure defined by pan-integral.

In the following we discuss some properties of $\lambda_{f}^{(\oplus, \otimes)}$ on the given pan-space $\left(X, \mathcal{A}, \mu, \bar{R}_{+}, \oplus, \otimes\right)$. We denote $\inf _{x \in X} f(x)$ by $\alpha_{f}$.

Proposition 9. ([6]) We have the following: $\forall A, B \in \mathcal{A}, f, g \in \mathcal{F}_{+}^{(X, \mathcal{A})}$,

1) $\lambda_{f}^{(\oplus, \otimes)}(A \cup B) \geq \lambda_{f}^{(\oplus, \otimes)}(A) \vee \lambda_{f}^{(\oplus, \otimes)}(B)$.

2) $\lambda_{f}^{(\oplus, \otimes)}(A \cap B) \leq \lambda_{f}^{(\oplus, \otimes)}(A) \wedge \lambda_{f}^{(\oplus, \otimes)}(B)$.

3) $\lambda_{f \vee g}^{(\oplus, \otimes)}(A) \geq \lambda_{f}^{(\oplus, \otimes)}(A) \vee \lambda_{g}^{(\oplus, \otimes)}(A)$.

4) $\lambda_{f \wedge g}^{(\oplus, \otimes)}(A) \leq \lambda_{f}^{(\oplus, \otimes)}(A) \wedge \lambda_{g}^{(\oplus, \otimes)}(A)$.

Proposition 10. We have the following:

1) $\lambda_{f}^{(\oplus, \otimes)} \ll_{I} \mu$, i.e., $\forall A \in \mathcal{A}$,

$$
\mu(A)=0 \text { implies } \lambda_{f}^{(\oplus, \otimes)}(A)=0 .
$$

Furthermore, if $\alpha_{f}>0$, i.e., $\inf _{x \in X} f(x)>0$, then for any $A \in \mathcal{A}$,

$$
\mu(A)=0 \text { if and only if } \lambda_{f}^{(\oplus, \otimes)}(A)=0 .
$$

2) If $\alpha_{f}>0$, then $\mu \ll_{V I} \lambda_{f}^{(\oplus, \otimes)}$, i.e., $\forall\left\{A_{n}\right\} \subset \mathcal{A}$, as $n \rightarrow \infty$,

$$
\lambda_{f}^{(\oplus, \otimes)}\left(A_{n}\right) \rightarrow 0 \text { implies } \mu\left(A_{n}\right) \rightarrow 0 \text {. }
$$

Proof. 1) From definition of the pan-integral, the (3.2) is obvious.

Let $\alpha_{f}>0$, then $\forall A \in \mathcal{A}$, if $\lambda_{f}^{(\oplus, \otimes)}(A)=0$, then 


$$
\lambda_{f}^{(\oplus, \otimes)}(A)=\int_{A}^{(\oplus, \otimes)} f \mathrm{~d} \mu \geq \int_{A}^{(\oplus, \otimes)} \alpha_{f} \mathrm{~d} \mu \geq \alpha_{f} \otimes \mu(A) .
$$

Therefore $\alpha_{f} \otimes \mu(A)=0$, and noting that $\alpha_{f}>0$, we get $\mu(A)=0$.

The proof of 2) is similar.

The following is an alternative statement of Proposition 6 2).

Proposition 11. Let $\mu$ be continuous from below and $A \in \mathcal{A}$. Then $f=0$ a.e. on $A$ if and only if $\lambda_{f}^{(\oplus, \otimes)}(A)=0$.

Proposition 12. Let $\alpha_{f}>0$. If $\mu$ is null-additive, then so is $\lambda_{f}^{(\oplus, \otimes)}$.

Proof. For any $A, B \in \mathcal{A}$ and $\lambda_{f}^{(\oplus, \otimes)}(B)=0$, by Proposition 101 ), we have $\mu(B)=0$. Noting that $f \chi_{A \cup B}=f \chi_{A}$ a.e. on $X$, from Proposition 63$)$ and 8 2) we have

$$
\begin{aligned}
\lambda_{f}^{(\oplus, \otimes)}(A \cup B) & =\int_{A \cup B}^{(\oplus, \otimes)} f \mathrm{~d} \mu=\int^{(\oplus, \otimes)} f \chi_{A \cup B} \mathrm{~d} \mu=\int^{(\oplus, \otimes)} f \chi_{A} \mathrm{~d} \mu \\
& =\int_{A}^{(\oplus, \otimes)} f \mathrm{~d} \mu=\lambda_{f}^{(\oplus, \otimes)}(A) .
\end{aligned}
$$

Similarly, we can get the following result.

Proposition 13. Let $\alpha_{f}>0$. If $\mu$ is weakly null-additive, then so is $\lambda_{f}^{(\oplus, \otimes)}$.

Proposition 14. If $\mu$ is continuous from below, then so is $\lambda_{f}^{(\oplus, \otimes)}$.

Proof. From Proposition 7, i.e., the Monotone Convergence Theorem of the pan-integral, and Proposition 63 ), we can obtain the desired conclusion.

We consider the Sugeno integral and Shilkret integral.

Proposition 15. 1) If $\mu$ is sub-additive, then so is $\lambda_{f}^{(v, \wedge)}$, that is, $\forall A, B \in \mathcal{A}$,

$$
\lambda_{f}^{(v, \wedge)}(A \cup B) \leq \lambda_{f}^{(\vee, \wedge)}(A)+\lambda_{f}^{(\vee, \wedge)}(B),
$$

i.e.,

$$
(S u) \int_{A \cup B} f \mathrm{~d} \mu \leq(S u) \int_{A} f \mathrm{~d} \mu+(S u) \int_{B} f \mathrm{~d} \mu .
$$

2) If $\mu$ is sub-additive, then so is $\lambda_{f}^{(v,)}$, that is, $\forall A, B \in \mathcal{A}$,

$$
\lambda_{f}^{(\mathrm{v},)}(A \cup B) \leq \lambda_{f}^{(\mathrm{v},)}(A)+\lambda_{f}^{(\mathrm{v},)}(B),
$$

i.e.,

$$
(S h) \int_{A \cup B} f \mathrm{~d} \mu \leq(S h) \int_{A} f \mathrm{~d} \mu+(S h) \int_{B} f \mathrm{~d} \mu .
$$

The following result was obtained in [14].

Proposition 16. We have the following:

1) If $\mu$ is $\vee$-additive (it is also called to be fuzzy additive, i.e., $\mu(P \cup Q)=\mu(P) \vee \mu(Q)$ for any $P, Q \in \mathcal{A})$, then so is $\lambda_{f}^{(\vee, \wedge)}$, that is, $\forall A, B \in \mathcal{A}$,

$$
\lambda_{f}^{(\vee, \wedge)}(A \cup B)=\lambda_{f}^{(\vee, \wedge)}(A) \vee \lambda_{f}^{(\vee, \wedge)}(B),
$$

i.e.,

$$
(S u) \int_{A \cup B} f \mathrm{~d} \mu=(S u) \int_{A} f \mathrm{~d} \mu \vee(S u) \int_{B} f \mathrm{~d} \mu .
$$

2) If $\mu$ is fuzzy multiplicative $(\mu(P \cap Q)=\mu(P) \wedge \mu(Q)$ for any $P, Q \in \mathcal{A})$, 
then so is $\lambda_{f}^{(v, \wedge)}$, that is, $\forall A, B \in \mathcal{A}$,

$$
\lambda_{f}^{(\vee, \wedge)}(A \cap B)=\lambda_{f}^{(\vee, \wedge)}(A) \wedge \lambda_{f}^{(v, \wedge)}(B),
$$

i.e.,

$$
(S u) \int_{A \cap B} f \mathrm{~d} \mu=(S u) \int_{A} f \mathrm{~d} \mu \wedge(S u) \int_{B} f \mathrm{~d} \mu .
$$

Similar to Proposition 16 we can get the following result for the Shilkret integral.

Proposition 17. We have the following:

1) If $\mu$ is $\vee$-additive, then so is $\lambda_{f}^{(v,)}$, that is, $\forall A, B \in \mathcal{A}$,

$$
\lambda_{f}^{(\vee,)}(A \cup B)=\lambda_{f}^{(\vee, \cdot)}(A) \vee \lambda_{f}^{(v, \cdot)}(B),
$$

i.e.,

$$
(S h) \int_{A \cup B} f \mathrm{~d} \mu=(S h) \int_{A} f \mathrm{~d} \mu \vee(S h) \int_{B} f \mathrm{~d} \mu
$$

2) If $\mu$ is fuzzy multiplicative, then so is $\lambda_{f}^{(v,)}$, that is, $\forall A, B \in \mathcal{A}$,

$$
\lambda_{f}^{(\vee, \wedge)}(A \cap B)=\lambda_{f}^{(\vee, *)}(A) \wedge \lambda_{f}^{(\vee, *)}(B),
$$

i.e.,

$$
(S h) \int_{A \cap B} f \mathrm{~d} \mu=(S h) \int_{A} f \mathrm{~d} \mu \wedge(S h) \int_{B} f \mathrm{~d} \mu .
$$

Definition 18. Let $\mu \in \mathcal{M}^{(X, \mathcal{A})}$, and $\left(X, \mathcal{A}, \mu, \bar{R}_{+}, \oplus, \otimes\right)$ be a pan-space. The monotone measure $\mu$ is said to be

1) sub- $\oplus$-additive, if $\mu(A \cup B) \leq \mu(A) \oplus \mu(B)$ whenever $A, B \in \mathcal{A}$;

2) super- $\oplus$-additive, if $\mu(A \cup B) \geq \mu(A) \oplus \mu(B)$ whenever $A, B \in \mathcal{A}$, $A \cap B=\varnothing$;

3) $\oplus$-additive, if $\mu(A \cup B)=\mu(A) \oplus \mu(B)$ for any $A, B \in \mathcal{A}, A \cap B=\varnothing$;

4) $\sigma$ - $\oplus$-additive, if for any disjoint sequence of sets $\left\{A_{n}\right\} \subset \mathcal{A}$,

$$
\mu\left(\bigcup_{n=1}^{\infty} A_{n}\right)=\bigoplus_{n=1}^{\infty} \mu\left(A_{n}\right)
$$

Proposition 19. Given the pan-space $\left(X, \mathcal{A}, \mu, \bar{R}_{+}, \oplus, \otimes\right)$ and $f \in \mathcal{F}_{+}^{(X, \mathcal{A})}$. If $\mu$ is sub- $\oplus$-additive, then so is $\lambda_{f}^{(\oplus, \otimes)}$.

Proof. For any $A, B \in \mathcal{A}$, we assume that $A \cap B=\varnothing$ without loss of generality. From Definition 5, we notice that $\mu$ is sub- $\oplus$-additive, thus

$$
\begin{aligned}
& \lambda_{f}^{(\oplus, \otimes)}(A \cup B)=\int_{A \cup B}^{(\oplus, \otimes)} f \mathrm{~d} \mu=\sup _{\mathcal{E} \in \mathcal{P}}\left\{\bigoplus_{E \in \mathcal{E}}\left[\left(\inf _{x \in(A \cup B) \cap E} f(x)\right) \otimes \mu((A \cup B) \cap E)\right]\right\} \\
& \leq \sup _{\mathcal{E} \in \mathcal{P}}\left\{\bigoplus_{E \in \mathcal{E}}\left[\left(\inf _{x \in(A \cup B) \cap E} f(x)\right) \otimes(\mu(A \cap E) \oplus \mu(B \cap E))\right]\right\} \\
& \leq \sup _{\mathcal{E} \in \mathcal{P}}\left\{\left\{\bigoplus_{E \in \mathcal{E}}\left[\left(\inf _{x \in A \cap E} f(x)\right) \otimes \mu(A \cap E)\right]\right\} \oplus\left\{\bigoplus_{E \in \mathcal{E}}\left[\left(\inf _{x \in B \cap E} f(x)\right) \otimes \mu(B \cap E)\right]\right\}\right\} \\
& \leq \sup _{\mathcal{E} \in \mathcal{P}}\left\{\bigoplus_{E \in \mathcal{E}}\left[\left(\inf _{x \in A \cap E} f(x)\right) \otimes \mu(A \cap E)\right]\right\} \oplus \sup _{\mathcal{E} \in \mathcal{P}}\left\{\bigoplus_{E \in \mathcal{E}}\left[\left(\inf _{x \in B \cap E} f(x)\right) \otimes \mu(B \cap E)\right]\right\} \\
& =\int^{(\oplus, \otimes)} f \chi_{A} \mathrm{~d} \mu \oplus \int^{(\oplus, \otimes)} f \chi_{B} \mathrm{~d} \mu=\lambda_{f}^{(\oplus, \otimes)}(A) \oplus \lambda_{f}^{(\oplus, \otimes)}(B) .
\end{aligned}
$$


The proof is complete.

We also have the following results (see [12]).

Proposition 20. Given the pan-space $\left(X, \mathcal{A}, \mu, \bar{R}_{+}, \oplus, \otimes\right)$ and $f \in \mathcal{F}_{+}^{(X, \mathcal{A})}$.

1) If $\mu$ is $\oplus$-additive, then so is $\lambda_{f}^{(\oplus, \otimes)}$.

2) If $\mu$ is $\sigma$-๑-additive, then so is $\lambda_{f}^{(\oplus, \otimes)}$.

Given the pan-space $\left(X, \mathcal{A}, \mu, \bar{R}_{+}, \oplus, \otimes\right)$, the pan-integral

$$
F^{(\oplus, \otimes)}(f)=\int^{(\oplus, \otimes)} f \mathrm{~d} \mu, \quad \forall f \in \mathcal{F}_{+}^{(X, \mathcal{A})}
$$

determines a monotone functional on $\mathcal{F}_{+}^{(X, \mathcal{A})}$.

In the following we show that if $\mu$ is sub- $\oplus$-additive, then the monotone functional $F^{(\oplus, \otimes)}$ is super- $\oplus$-additive.

Proposition 21. Given the pan-space $\left(X, \mathcal{A}, \mu, \bar{R}_{+}, \oplus, \otimes\right)$. If $\mu$ is sub- $\oplus$-additive, then for any $f, g \in \mathcal{F}_{+}^{(X, \mathcal{A})}$, it holds

$$
F^{(\oplus, \otimes)}(f \oplus g) \geq F^{(\oplus, \otimes)}(f) \oplus F^{(\oplus, \otimes)}(g),
$$

i.e.,

$$
\int^{(\oplus, \otimes)}(f \oplus g) \mathrm{d} \mu \geq \int^{(\oplus, \otimes)} f \mathrm{~d} \mu \oplus \int^{(\oplus, \otimes)} g \mathrm{~d} \mu .
$$

Proof: Let $f, g \in \mathcal{F}_{+}^{(X, \mathcal{A})}$. For any

$$
\bigoplus_{i=1}^{r}\left(\lambda_{i} \otimes \chi_{A_{i}}\right) \leq f \text { and } \bigoplus_{j=1}^{k}\left(\delta_{j} \otimes \chi_{B_{j}}\right) \leq g,
$$

where $\lambda_{i} \geq 0, \delta_{j} \geq 0, i=1,2, \cdots, r, j=1,2, \cdots, k,\left\{A_{i}\right\}_{i=1}^{r} \in \hat{\mathcal{P}},\left\{B_{j}\right\}_{j=1}^{k} \in \hat{\mathcal{P}}$, we have

$$
\left(\bigoplus_{i=1}^{r}\left(\lambda_{i} \otimes \chi_{A_{i}}\right)\right) \oplus\left(\bigoplus_{j=1}^{k}\left(\delta_{j} \otimes \chi_{B_{j}}\right)\right) \leq f \oplus g .
$$

On the other hand,

$$
\bigoplus_{i=1}^{r}\left\{\bigoplus_{j=1}^{k}\left(\left(\lambda_{i} \oplus \delta_{j}\right) \otimes \chi_{A_{i} \cap B_{j}}\right)\right\}=\left(\bigoplus_{i=1}^{r}\left(\lambda_{i} \otimes \chi_{A_{i}}\right)\right) \oplus\left(\bigoplus_{j=1}^{k}\left(\delta_{j} \otimes \chi_{B_{j}}\right)\right) .
$$

Noting that $\left\{A_{i} \cap B_{j}: i=1,2, \cdots, r, j=1,2, \cdots, k\right\} \in \hat{\mathcal{P}}$, it follows

$$
\bigoplus_{i=1}^{r} \bigoplus_{j=1}^{k}\left(\left(\lambda_{i} \oplus \delta_{j}\right) \otimes \mu\left(A_{i} \cap B_{j}\right)\right) \leq \int(f \oplus g) \mathrm{d} \mu \text {. }
$$

Since $\mu$ is sub- $\oplus$-additive, we have

$$
\begin{aligned}
& \left(\bigoplus_{i=1}^{r}\left(\lambda_{i} \otimes \mu\left(A_{i}\right)\right)\right) \oplus\left(\bigoplus_{j=1}^{k}\left(\lambda_{j} \otimes \mu\left(B_{j}\right)\right)\right) \\
& \leq\left\{\bigoplus_{i=1}^{r}\left(\lambda_{i} \otimes \bigoplus_{j=1}^{k}\left[\mu\left(A_{i} \cap B_{j}\right)\right]\right)\right\} \oplus\left\{\bigoplus_{j=1}^{k}\left(\delta_{j} \otimes \bigoplus_{i=1}^{r}\left[\mu\left(A_{i} \cap B_{j}\right)\right]\right)\right\} \\
& \leq \bigoplus_{i=1}^{r} \bigoplus_{j=1}^{k}\left(\left(\lambda_{i} \oplus \delta_{j}\right) \otimes \mu\left(A_{i} \cap B_{j}\right)\right) \\
& \leq \int^{(\oplus, \otimes)}(f \oplus g) \mathrm{d} \mu .
\end{aligned}
$$

Therefore,

$$
\int^{(\oplus, \otimes)} f \mathrm{~d} \mu \oplus \int^{(\oplus, \otimes)} g \mathrm{~d} \mu \leq \int^{(\oplus, \otimes)}(f \oplus g) \mathrm{d} \mu .
$$


The proof is complete.

In the special case of the usual addition + and multiplication, the following results have been obtained in [25]:

Proposition 22. Given the pan-space $\left(X, \mathcal{A}, \mu, \bar{R}_{+},+, \cdot\right)$ and $f \in \mathcal{F}_{+}^{(X, \mathcal{A})}$. If $\mu$ is subadditive, then so is $\lambda_{f}^{(\oplus, \otimes)}$, i.e., any $A, B \in \mathcal{A}$,

$$
\int_{A \cup B}^{(+,)} f \mathrm{~d} \mu=\int_{A}^{(+,)} f \mathrm{~d} \mu+\int_{B}^{(+,)} f \mathrm{~d} \mu .
$$

Proposition 23. Given the pan-space $\left(X, \mathcal{A}, \mu, \bar{R}_{+},+, \cdot\right)$ If $\mu$ is subadditive, then for any $f, g \in \mathcal{F}_{+}^{(X, \mathcal{A})}$, it holds

$$
F^{(+,)}(f+g)=F^{(+,)}(f)+F^{(+,)}(g),
$$

i.e.,

$$
\int^{(+,)}(f+g) \mathrm{d} \mu=\int^{(+,)} f \mathrm{~d} \mu+\int^{(+,)} g \mathrm{~d} \mu .
$$

\section{Conclusions}

We have discussed the monotone measures defined by the pan-integral (with respect to monotone measure $\mu$ and operations $(\oplus, \otimes)$ ). As what has been discussed above, this construction inherited almost all desirable structural characteristics of the original monotone measure $\mu$. They are continuity from below, $\oplus$-additivity, $\oplus$-subadditivity, null-additivity, weak null-additivity, etc. We have also shown that the pan-integral with respect to the subadditive monotone measure is monotone superadditive functional.

In general, monotone measures lose additivity, they appear to be much looser than the classical measures. Thus, it is quite difficult to develop a general theory of monotone measures and nonlinear integral without any additional condition. The concepts mentioned above replace additivity play important roles in generalized measure theory (see [6] [18] [19] [26] [27] [28]). So, the results we obtained here are significant when applying monotone measure theory to expert systems, decision making, pattern recognition, image processing, risk analysis, and other areas.

The concave integral is a type of important nonlinear integral (with respect to capacity) [4] [29]. In further research, we will investigate properties of monotone measures defined by concave integral.

We point out that there are three important structural characteristics of monotone set function: strong order continuity [26] (see also [30]), the property (S) and the condition (E) [27]. It has been shown that Lebesgue's theorem, Riesz's theorem and Egoroffs theorem in classical measures theory ([20] [31]) hold in the case of monotone measures if and only if the monotone measures possess strong order continuity [26] [27] [28] (see also [30]). We state these three concepts in the following:

Definition 24. ([26]) A monotone measure $\mu: \mathcal{A} \rightarrow[0,+\infty]$ is said to be strongly order continuous, if $\lim _{n \rightarrow \infty} \mu\left(P_{n}\right)=0$ whenever $P_{n} \searrow N$ with $\mu(N)=0$. 
Definition 25. ([28] A fuzzy measure $\mu$ is said to have property) (S), if for any $\left\{A_{n}\right\}_{n}$ with $\lim _{n \rightarrow \infty} \mu\left(A_{n}\right)=0$, there exists a subsequence $\left\{A_{n_{i}}\right\}_{i}$ of $\left\{A_{n}\right\}_{n}$ such that

$$
\mu\left(\bigcap_{k=1}^{\infty} \bigcup_{i=k}^{\infty} A_{n_{i}}\right)=0
$$

Definition 26. ([27]) A fuzzy measure $\mu$ is said to fulfill the condition $(E)$, if for every double sequence $\left\{A_{n}^{(k)} \mid n \geq 1, k \geq 1\right\} \subset \mathcal{A}$ satisfying the conditions: for fixed $n=1,2, \cdots$,

$$
A_{n}^{(k)} \searrow D_{n}(k \rightarrow \infty) \text { and } \mu\left(\bigcup_{n=1}^{\infty} D_{n}\right)=0,
$$

there exist increasing sequences $\left\{k_{i}\right\}_{i \in \mathbb{N}}$ and $\left\{n_{i}\right\}_{i \in \mathbb{N}}$ of natural numbers, such that

$$
\lim _{j \rightarrow+\infty} \mu\left(\bigcup_{i=j}^{\infty} A_{n_{i}}^{\left(k_{i}\right)}\right)=0
$$

We don't know whether the monotone measure $\lambda_{f}^{(\oplus, \otimes)}$ defined by the pan-integral with respect to monotone measure $\mu$ and operations $(\oplus, \otimes)$ can inherit these three important structural characteristics of monotone measures (even in special cases of $(\vee, \wedge)$ or $(\vee, \cdot)$, it is not clear). We will further investigate these problems.

\section{Acknowledgements}

This research was partially supported by the National Natural Science Foundation of China (Grant No. 11571106).

\section{References}

[1] Choquet, G. (1953) Theory of Capacitie. Annales de l'Institut Fourier, 5, 131-295. https://doi.org/10.5802/aif.53

[2] Sugeno, M. (1974) Theory of Fuzzy Integrals and Its Application. Ph. D. Thesis, Tokyo Institute of Technology, Tokyo.

[3] Yang, Q. (1985) The Pan-Integral on Fuzzy Measure Space. Fuzzy Mathematics, 3, 107-114. (In Chinese)

[4] Lehrer, E. and Teper, R. (2008) The Concave Integral over Large Spaces. Fuzzy Sets and Systems, 159, 2130-2144. https://doi.org/10.1016/j.fss.2007.11.018

[5] Klement, E.P., Li, J., Mesiar, R. and Pap, E. (2015) Integrals Based on Monotone Set Functions. Fuzzy Sets and Systems, 281, 88-102. https://doi.org/10.1016/j.fss.2015.07.010

[6] Wang, Z. and Klir, G.J. (2009) Generalized Measure Theory. Springer, Berlin. https://doi.org/10.1007/978-0-387-76852-6

[7] Li, J., Ouyang, Y. and Yu, M. (2017) Pan-Integrals Based on Optimal Measure. Lecture Notes in Computer Science, 10571, 30-40. https://doi.org/10.1007/978-3-319-62024-4_3

[8] Mesiar, R. and Rybárik, J. (1995) Pan-Operations Structure. Fuzzy Sets and Systems, 74, 365-369. https://doi.org/10.1016/0165-0114(94)00314-W 
[9] Ouyang, Y. and Li, J. (2016) An Equivalent Definition of the Pan-Integral. Lecture Notes in Computer Science, 9880, 107-113. https://doi.org/10.1007/978-3-319-45656-0_9

[10] Pap, E. (1995) Null-Additive Set Functions. Kluwer, Dordrecht.

[11] Sugeno, M. and Murofushi, T. (1987) Pseudo-Additive Measures and Integrals. Journal of Mathematical Analysis and Applications, 122, 197-222. https://doi.org/10.1016/0022-247X(87)90354-4

[12] Zhang, Q., Li, J., Mesiar, R. and Struk, P. (2011) Generalized Lebesgue Integral. International Journal of Approximate Reasoning, 52, 427-443. https://doi.org/10.1016/j.ijar.2010.10.002

[13] Bogachev, V.L. (2006) Measure Theory. Springer, Berlin.

[14] Wang, Z., Klir, G.J. and Harmanec, D. (1995) The Preservation of Structural Characteristics of Monotone Set Functions Defined by Fuzzy Integral. Journal of Fuzzy Mathematics, 3, 229-240.

[15] Wang, Z., Klir, G.J. and Wang, W. (1996) Monotone Set Functions Defined by Choquet Integral. Fuzzy Sets and Systems, 81, 241-250. https://doi.org/10.1016/0165-0114(95)00181-6

[16] Ouyang, Y. and Li, J. (2004) A Note on the Monotone Set Function Defined by Choquet Integral. Fuzzy Sets and Systems, 146, 147-151.

https://doi.org/10.1016/j.fss.2003.12.008

[17] Benvenuti, P., Mesiar, R. and Vivona, D. (2002) Monotone Set Functions-Based Integrals. In: Pap, E., Ed., Handbook of Measure Theory, Elsevier, Amsterdam, 1329-1379. https://doi.org/10.1016/B978-044450263-6/50034-8

[18] Denneberg, D. (1994) Non-Additive Measure and Integral. Springer, Berlin. https://doi.org/10.1007/978-94-017-2434-0

[19] Torra, V., Narukawa, Y. and Sugeno, M. (2014) Non-Additive Measure-Theory and Applications. Studies in Fuzziness and Soft Computing, Vol. 310, Springer, Berlin.

[20] Halmos, P.R. (1968) Measure Theory. Van Nostrand, New York.

[21] Li, J., Mesiar, R. and Zhang, Q. (2010) Absolute Continuity of Monotone Measure and Convergence in Measure. Communications in Computer and Information Science, 80, 500-504. https://doi.org/10.1007/978-3-642-14055-6_52

[22] Wang, Z., Klir, G.J. and Wang, W. (1996) Fuzzy Measures Defined by Fuzzy Integral and Their Absolute Continuity. Journal of Mathematical Analysis and Applications, 203, 150-165. https://doi.org/10.1006/jmaa.1996.0372

[23] Benvenuti, P. and Mesiar, R. (2004) Pseudo-Arithmetical Operations as a Basis for the General Measure and Integration Theory. Information Sciences, 160, 1-11. https://doi.org/10.1016/j.ins.2003.07.005

[24] Shilkret, N. (1971) Maxitive Measure and Integration. Indagationes Mathematicae, 33, 109-116. https://doi.org/10.1016/S1385-7258(71)80017-3

[25] Ouyang, Y., Li, J. and Mesiar, R. (2017) On Linearity of Pan-Integral and Pan-Integrable Functions Space. International Journal of Approximate Reasoning, 90, 307-318. https://doi.org/10.1016/j.ijar.2017.08.001

[26] Li, J. (2003) Order Continuity of Monotone Set Function and Convergence of Measurable Functions Sequence. Applied Mathematics and Computation, 135, 211-218. https://doi.org/10.1016/S0096-3003(01)00317-4

[27] Li, J. and Yasuda, M. (2005) On Egoroff's Theorems on Finite Monotone 
Non-Additive Measure Spaces. Fuzzy Sets and Systems, 153, 71-78. https://doi.org/10.1016/j.fss.2005.01.010

[28] Sun, Q. (1994) Property (S) of Fuzzy Measure and Riesz's Theorem. Fuzzy Sets and Systems, 62, 117-119. https://doi.org/10.1016/0165-0114(94)90080-9

[29] Mesiar, R., Li, J. and Pap, E. (2011) Pseudo-Concave Integrals. Advances in Intelligent Systems and Computing, 100, 43-49.

[30] Li, J., Mesiar, R., Pap, E. and Klement, E.P. (2015) Convergence Theorems for Monotone Measures. Fuzzy Sets and Systems, 281, 103-127. https://doi.org/10.1016/j.fss.2015.05.017

[31] Hewitt, E. and Stromberg, K. (1965) Real and Abstract Analysis. Springer, New York. 